\title{
Impact of Omitting Fluorouracil From FOLFIRI Plus Bevacizumab as Second-line Chemotherapy for Patients With Metastatic Colorectal Cancer
}

\section{Yuki Matsubara}

Aichi-ken Gan Center

Toshiki Masuishi ( $\square$ tmasuishi@aichi-cc.jp )

Aichi-ken Gan Center https://orcid.org/0000-0002-7521-1596

\section{Takatsugu Ogata}

Aichi-ken Gan Center

\section{Taiko Nakazawa}

Aichi-ken Gan Center

Kyoko Kato

Aichi-ken Gan Center

Kazuki Nozawa

Aichi-ken Gan Center

Yukiya Narita

Aichi-ken Gan Center

Kazunori Honda

Aichi-ken Gan Center

Hideaki Bando

Aichi-ken Gan Center

Hiroya Taniguchi

Aichi-ken Gan Center

Shigenori Kadowaki

Aichi-ken Gan Center

Masashi Ando

Aichi-ken Gan Center

Masahiro Tajika

Aichi-ken Gan Center

Kei Muro

Aichi-ken Gan Center 
Keywords: Colorectal Cancer, Chemotherapy, Fluoropyrimidine, Irinotecan, Bevacizumab

Posted Date: February 4th, 2022

DOI: https://doi.org/10.21203/rs.3.rs-1309620/v1

License: (c) (1) This work is licensed under a Creative Commons Attribution 4.0 International License. Read Full License

Version of Record: A version of this preprint was published at Journal of Clinical Oncology on January 20th, 2021. See the published version at https://doi.org/10.1200/JC0.2021.39.3_suppl.76. 


\section{Abstract}

\section{Purpose}

Fluorouracil, leucovorin, and irinotecan (FOLFIRI) plus bevacizumab is the standard second-line chemotherapy for patients with metastatic colorectal cancer (mCRC) who are refractory or intolerant to fluoropyrimidines and oxaliplatin. However, the benefits of incorporating fluoropyrimidines into secondline chemotherapy for patients with $\mathrm{mCRC}$ who are refractory to fluoropyrimidines in first-line chemotherapy are unknown.

\section{Methods}

We retrospectively evaluated patients with $\mathrm{mCRC}$ who were administered irinotecan plus bevacizumab or FOLFIRI plus bevacizumab as second-line chemotherapy at a single institution from January 2010 to April 2020. We compared the efficacy and safety of irinotecan plus bevacizumab (IRI group) with those of FOLFIRI plus bevacizumab (FOLFIRI group).

\section{Results}

Of the 255 enrolled patients, 107 (IRI/FOLFIRI group, 31/76 patients) were eligible for analysis. After a median follow-up of 13.1 months (range, 1.2-48.4) and 14.3 months (range, 0.9-46.5) for the IRI and FOLFIRI groups, respectively, the median progression-free survival was 6.4 months and 5.8 months [adjusted hazard ratio (aHR), 0.82; 95\% confidence interval (Cl), 0.50-1.34, $p=0.44$ ] and the median overall survival was 16.6 months and 16.5 months (aHR, $1.01 ; 95 \% \mathrm{Cl}, 0.59-1.69 ; p=0.97$ ) in the IRI and FOLFIRI groups, respectively. All grade nausea, vomiting, decreased appetite, stomatitis, hand-foot syndrome, neutropenia, thrombocytopenia, increased creatinine, Grade 3/4 neutropenia, and febrile neutropenia occurred more frequently in the FOLFIRI group than in the IRI group.

\section{Conclusion}

Omitting fluorouracil from FOLFIRI plus bevacizumab as second-line chemotherapy may reduce adverse events without affecting antitumor activity in patients with $\mathrm{MCRC}$ who are refractory to fluoropyrimidines.

\section{Introduction}

Fluorouracil (5-FU)/leucovorin (LV) plus irinotecan (FOLFIRI) combined with bevacizumab (antivascular endothelial growth factor [VEGF]) is a standard second-line chemotherapeutic regimen with metastatic colorectal cancer (mCRC) patients who are refractory or intolerant to fluoropyrimidines plus oxaliplatin as first-line treatment. This regimen is supported by the results of the GERCOR V308 phase 3 study, which showed a similar efficacy between 5-FU/LV plus oxaliplatin (FOLFOX) followed by FOLFIRI and FOLFIRI followed by FOLFOX (Tournigand et al. 2004). For patients with mCRC who were refractory to irinotecan and bolus 5-FU/LV, FOLFOX was superior to single-agent oxaliplatin (Rothenberg et al. 2003). However, it is unclear whether the addition of 5-FU to irinotecan enhances efficacy in patients with mCRC who are 
refractory to 5-FU. Moreover, continuing fluoropyrimidine therapy in patients with $\mathrm{mCRC}$ who progressed after standard first-line fluoropyrimidines-based treatment may only result in increased side effects. In this study, we retrospectively evaluated the effect of omitting 5-FU from FOLFIRI plus bevacizumab as second-line chemotherapy for patients with $\mathrm{MCRC}$ who were refractory to fluoropyrimidines.

\section{Patients And Methods}

\section{Patients}

We retrospectively reviewed data for patients with $\mathrm{mCRC}$ who received irinotecan plus bevacizumab (IRI group) or FOLFIRI plus bevacizumab (FOLFIRI group) as second-line chemotherapy after first-line treatment with fluoropyrimidine and oxaliplatin-based chemotherapy at the Aichi Cancer Center Hospital. The inclusion criteria were as follows: (1) second-line chemotherapy initiated from January 2010 to April 2020, (2) Eastern Cooperative Oncology Group Performance Status (ECOG PS) of 0-2, (3) pathological diagnosis of colorectal adenocarcinoma, (4) KRAS status, (5) refractory to fluoropyrimidines, (6) refractory or intolerant to oxaliplatin, (7) no prior treatment with irinotecan, (8) CT scan within 28 days before initiation of second-line chemotherapy and receipt of a standard dose of each drug. Patients in the IRI group received an intravenous infusion of irinotecan of $150 \mathrm{mg} / \mathrm{m}^{2}$ and bevacizumab of $5 \mathrm{mg} / \mathrm{kg}$ on day 1 , every 2 weeks. Patients in the FOLFIRI group received intravenous infusion of Heucovorin isomers of $200 \mathrm{mg} / \mathrm{m}^{2}$, a bolus of $5-\mathrm{FU}$ of $400 \mathrm{mg} / \mathrm{m}^{2}$, a $46-\mathrm{h}$ continuous infusion of $5-\mathrm{FU}$ at $2,400 \mathrm{mg} / \mathrm{m}^{2}$, irinotecan at $150 \mathrm{mg} / \mathrm{m}^{2}$, and bevacizumab at $5 \mathrm{mg} / \mathrm{kg}$ on day 1 , every 2 weeks. Resistance to fluoropyrimidines was defined as disease progression during or within 8 weeks of the last dose of firstline fluoropyrimidine therapy, or relapse within 6 months of the last dose of adjuvant fluoropyrimidine therapy. Previous use of bevacizumab as first-line treatment was permitted. Oxaliplatin-containing adjuvant chemotherapeutic regimens were regarded as first-line treatment if relapse occurred within 6 months from the last dose of oxaliplatin. In contrast, fluoropyrimidines alone as adjuvant chemotherapy were not considered first-line treatment in any case. All the patients provided written informed consent for receiving chemotherapy. This study was approved by the Institutional Review Board of the Aichi Cancer Center Hospital (2020-1-339) and conducted in accordance with the Declaration of Helsinki.

\section{Outcomes and statistical analysis}

We reviewed medical records and collected patient characteristics, pathological features, and treatment history with a cutoff date of May 31,2020. Progression-free survival (PFS) and overall survival (OS) were defined as the time from the date of initiation of second-line treatment to the date of disease progression, or death from any cause and to the date of death, respectively. Tumor response was assessed according to Response Evaluation Criteria in Solid Tumors version 1.1 (RECIST v1.1). Adverse events were assessed during second-line chemotherapy according to Common Terminology Criteria for Adverse Events version 5.0. PFS and OS were estimated using the Kaplan-Meier method. The adjusted hazard ratios (aHRs) for PFS and OS were calculated using a multivariate Cox proportional hazards model and contained 
variables with $p$ values $<0.05$ in a univariate analysis to reduce the imbalance between groups. Variables included in the univariate and multivariate analyses were as follows: age ( $<65 \mathrm{vs.} \geq 65$ ), sex (male vs. female), KRAS (wild-type vs. mutant), BRAF V600E (wild-type vs. mutant), histology (well- or moderatelydifferentiated adenocarcinoma vs. poorly-differentiated adenocarcinoma, signet-ring cell carcinoma, or mucinous carcinoma), previous colorectal resection (yes vs. no), primary tumor location (right-sided vs. left-sided), the number of metastatic sites ( $1-2$ vs. $\geq 3$ ), first-line PFS ( $<6$ months vs. $\geq 6$ months), previous fluoropyrimidine therapy (capecitabine or S-1 vs. fluorouracil), previous use of bevacizumab in first-line treatment (yes vs. no), ECOG PS (0 vs. 1-2), liver metastasis (yes vs. no), lung metastasis (yes vs. no), peritoneal metastasis (yes vs. no), alkaline phosphatase (ALP) levels ( $<300 \mathrm{vs.} \geq 300 \mathrm{U} / \mathrm{L}$ ), and lactate dehydrogenase ( $\mathrm{LDH}$ ) levels ( $<400 \mathrm{vs.} \geq 400 \mathrm{U} / \mathrm{L}$ ). Right-sided tumors arise from the cecum, ascending colon, and transverse colon, whereas left-sided tumors arise from the descending colon, sigmoid colon, and rectum. In addition, subgroup analyses according to these factors were assessed with a Cox proportional hazards model to estimate HRs for treatment effect. We assessed the difference in response rate $(R R)$, disease control rate $(D C R)$, and the proportion of patients with $\mathrm{mCRC}$ who were treated with third-line chemotherapy using Fisher's exact tests. The difference in BRAF V600E status and histology was analyzed by Pearson's Chi-square tests and the other characteristics were evaluated using Fisher's exact tests. In addition, deepness of response (DpR) was defined as the rate of tumor shrinkage from baseline $\mathrm{CT}$. The relative dose intensity (RDI) of each drug was compared between the two treatment groups using an analysis of variance. Differences in $p$ values $<0.05$ were considered statistically significant. The statistical analyses were performed using JMP® 10 software (SAS Institute Inc., Cary, NC, USA).

\section{Results}

\section{Patients}

From January 2010 to April 2020, 255 patients with mCRC received irinotecan plus bevacizumab or FOLFIRI plus bevacizumab as second-line chemotherapy after first-line treatment with fluoropyrimidines plus oxaliplatin at the Aichi Cancer Center Hospital. Of the 107 eligible patients, 31 received irinotecan plus bevacizumab (IRI group) and 76 received FOLFIRI plus bevacizumab (FOLFIRI group). The reasons for exclusion were as follows: initial dose reduction of irinotecan or 5-FU $(n=119)$, unknown KRAS status $(n=3)$, over 28 days from the last CT evaluation $(n=22)$, not refractory to 5 -FU $(n=1)$, simultaneous triple colorectal cancers $(n=1)$, change of regimen during first- or second-line treatment before disease progression $(n=1)$, unknown details regarding first-line treatment $(n=1)$.

The baseline characteristics of the patients are shown in Table 1. The patients in the IRI group contained more patients who received oral fluoropyrimidines and bevacizumab in first-line chemotherapy compared with the FOLFIRI group ( $65 \%$ vs. $34 \% ; p=0.005$, and $84 \%$ vs. $63 \% ; p=0.04$, respectively). On the other hand, fewer patients in the IRI group had right-sided tumors (19\% vs. $43 \%, p=0.03)$ and liver metastases ( $42 \%$ vs. $62 \%$ ) compared with those in the FOLFIRI group. Both groups exhibited a similar proportion of the other characteristics. 
Table 1

Patient characteristics

Characteristics

\begin{tabular}{ll} 
IRI group & FOLFIR \\
\hline $\mathrm{n}=\mathbf{3 1}$ & $\mathrm{n}=\mathbf{7 6}$ \\
$\mathrm{n}(\%)$ & $\mathrm{n}(\%)$ \\
\hline
\end{tabular}

Age

median (margin)
$<65$
$\geq 65$

Sex

$\begin{array}{lll}\text { Male } & 21(68) & 48(63) \\ \text { Female } & 10(32) & 28(37)\end{array}$

KRAS status

Wild-type

Mutant

BRAF V600E status

Wild-type

$27(87)$

2 (6)

2 (6)

$17(55)$

$14(45)$

Mutant

Unknown

Histology

well, mod
por, sig, muc
Unknown

Previous colorectal resection

Yes

$5(16)$

$26(84)$

No

Primary location

$\begin{array}{ll}22(71) & 52(68) \\ 8(26) & 24(32) \\ 1(3) & 0(0)\end{array}$

24 (32)

$52(68)$
0.13

62 (34-76)

$48(63)$

$28(37)$

0.82

0.68

$38(50)$

$38(50)$

0.70

$64(84)$

$8(11)$

$4(5)$

0.26

0.15

Abbreviations: Well, well-differentiated adenocarcinoma; mod, moderately-differentiated adenocarcinoma; por, poorly-differentiated adenocarcinoma; sig, signet-ring cell carcinoma; muc, mucinous carcinoma; PFS, progression-free survival; ECOG PS, Eastern Cooperative Oncology Group Performance Status; ALP, alkaline phosphatase; LDH, lactate dehydrogenase. 


\section{Characteristics}

Right-sided

Left-sided

Metastatic sites

$$
\begin{aligned}
& 1-2 \\
& \geq 3
\end{aligned}
$$

First-line PFS

$$
\begin{aligned}
& <6 \text { months } \\
& \geq 6 \text { months }
\end{aligned}
$$

Previous fluoropyrimidine

Capecitabine or S-1

Fluorouracil

Previous bevacizumab

Yes
No

ECOG PS

0

1,2

Liver metastasis

$\begin{array}{ll}\text { Yes } & 13(42) \\ \text { No } & 18(58)\end{array}$

Lung metastasis

\begin{tabular}{lll} 
Yes & $17(55)$ & $37(49)$ \\
\hline No & $14(45)$ & $39(51)$
\end{tabular}

Abbreviations: Well, well-differentiated adenocarcinoma; mod, moderately-differentiated adenocarcinoma; por, poorly-differentiated adenocarcinoma; sig, signet-ring cell carcinoma; muc, mucinous carcinoma; PFS, progression-free survival; ECOG PS, Eastern Cooperative Oncology Group Performance Status; ALP, alkaline phosphatase; LDH, lactate dehydrogenase.

0.42

0.50

0.005

0.04

0.67

$\begin{array}{ll}14(45) & 41(54) \\ 17(55) & 35(46)\end{array}$

$47(62)$

29 (38) 


\begin{tabular}{|c|c|c|c|}
\hline \multirow[t]{3}{*}{ Characteristics } & IRI group & FOLFIRI group & \multirow[t]{3}{*}{$p$ value } \\
\hline & $\mathrm{n}=31$ & $\mathrm{n}=76$ & \\
\hline & n (\%) & $\mathrm{n}(\%)$ & \\
\hline \multicolumn{3}{|l|}{ Peritoneal metastasis } & \multirow[t]{3}{*}{0.82} \\
\hline Yes & $9(29)$ & $25(33)$ & \\
\hline No & $22(71)$ & $51(67)$ & \\
\hline \multicolumn{3}{|l|}{ ALP } & \multirow[t]{4}{*}{0.52} \\
\hline$<300 \mathrm{U} / \mathrm{L}$ & $15(48)$ & $30(40)$ & \\
\hline$\geq 300 \mathrm{U} / \mathrm{L}$ & $16(52)$ & $45(59)$ & \\
\hline Unknown & $0(0)$ & $1(1)$ & \\
\hline \multicolumn{3}{|l|}{ LDH } & \multirow[t]{2}{*}{1.00} \\
\hline$<400 \mathrm{U} / \mathrm{L}$ & $26(84)$ & $65(86)$ & \\
\hline$\geq 400 \mathrm{U} / \mathrm{L}$ & $4(13)$ & $9(12)$ & \\
\hline Unknown & $1(3)$ & $2(3)$ & \\
\hline \multicolumn{4}{|c|}{$\begin{array}{l}\text { Abbreviations: Well, well-differentiated adenocarcinoma; mod, moderately-differentiated } \\
\text { adenocarcinoma; por, poorly-differentiated adenocarcinoma; sig, signet-ring cell carcinoma; muc, } \\
\text { mucinous carcinoma; PFS, progression-free survival; ECOG PS, Eastern Cooperative Oncology Group } \\
\text { Performance Status; ALP, alkaline phosphatase; LDH, lactate dehydrogenase. }\end{array}$} \\
\hline
\end{tabular}

Median follow-up time was 13.1 months (range, 1.2-48.4) in the IRI group and 14.3 months (range, 0.946.5) in the FOLFIRI group. On May 31, 2020, 100 patients (93\%) experienced disease progression during second-line treatment, which included 29 patients (94\%) in the IRI group and 71 patients (93\%) in the FOLFIRI group. After disease progression following second-line treatment, 18 patients $(62 \%)$ and 52 patients $(73 \%)$ received third-line chemotherapy in the IRI group and in the FOLFIRI group, respectively ( $p$ $=0.50$ ), which consisted of trifluridine/tipiracil plus bevacizumab (IRI group vs. FOLFIRI group, 33\% vs. $25 \%)$, regorafenib ( $6 \%$ vs. $6 \%$ ), irinotecan plus panitumumab or cetuximab ( $39 \%$ vs. $36 \%)$, or other treatment regimens (22\% vs. $33 \%)$.

\section{Efficacy}

PFS in the IRI group was similar to that in the FOLFIRI group [median PFS, 6.4 months vs. 5.8 months; hazard ratio (HR), 0.90; $95 \%$ confidence interval $(\mathrm{Cl}), 0.57-1.38 ; p=0.64]$ (Figure 1). Adjusted HR for PFS was $0.82(95 \% \mathrm{Cl}, 0.50-1.34 ; p=0.44)$ with covariates of previous colorectal resection, number of metastatic sites, ECOG PS, liver metastasis, and LDH levels. 
OS in the IRI group was similar to that in the FOLFIRI group (median OS, 16.6 vs. 16.5 months; HR, 0.83; $95 \% \mathrm{Cl}, 0.51-1.32 ; p=0.44)$ (Figure 1). Adjusted HR for PFS was $1.01(95 \% \mathrm{Cl}, 0.59-1.69 ; p=0.97)$ with the covariates of previous colorectal resection, number of metastatic sites, liver metastasis, peritoneal metastasis, and LDH levels. The results of the subgroup analyses for PFS and OS according to the patient characteristics were consistent with those of the entire population.

Among the patients with measurable lesions, RR was $26 \%$ in the IRI group and $11 \%$ in the FOLFIRI group. $(p=0.12$ ). DCR was $72 \%$ in the IRI group and $71 \%$ in the FOLFIRI group $(p=1.00)$ (Table 2$)$. DpR in the IRI group (median, $-10.3 \%$; range, $-74-30 \%$ ) was similar to that in the FOLFIRI group (median, $0 \%$; range, $-100-129 \%)$ (Figure 2).

Table 2

Relative dose intensity

\begin{tabular}{|llll|}
\hline Drug & \multicolumn{2}{l}{ IRI group } & \multicolumn{2}{l}{ FOLFIRI group } & \multirow{2}{*}{ p value } \\
\cline { 2 - 3 } & $\mathbf{n = 3 1}$ & $\mathbf{n}=76$ & \\
\cline { 2 - 3 } & $\%$ (range) & $\%$ (range) & \\
\hline Bolus 5-FU & - & $66(2-100)$ & - \\
\hline Infusional 5-FU & - & $83(20-100)$ & - \\
\hline Irinotecan & $80(44-100)$ & $82(26-100)$ & 0.633 \\
\hline Bevacizumab & $86(35-100)$ & $83(20-100)$ & 0.619 \\
\hline Abbreviation; 5-FU, fluorouracil; IRI, irinotecan; BEV, bevacizumab \\
\hline
\end{tabular}

\section{RDI}

RDI of irinotecan and bevacizumab was similar in the IRI and FOLFIRI groups [median (range) RDI of irinotecan, $80(44-100)$ vs. $82(26-100) \%(p=0.63)$; median RDI of bevacizumab, $86(35-100)$ vs. 83 $(20-100) \%(p=0.62)]$. Additionally, the median RDI of bolus and infusional 5-FU was $66(2-100) \%$ and $83(20-100) \%$, respectively (Table 2$)$.

\section{Safety}

All grade nausea (IRI group vs. FOLFIRI group, $32 \%$ vs. $58 \%, p=0.02)$, vomiting ( $13 \%$ vs. $28 \%, p=0.09$ ), decreased appetite ( $36 \%$ vs. $51 \%, p=0.14)$, stomatitis ( $13 \%$ vs. $36 \%, p=0.02)$, hand-foot syndrome ( $0 \%$ vs. $14 \%, p=0.06$ ), neutropenia ( $42 \%$ vs. $78 \%, p<0.001$ ), thrombocytopenia ( $0 \%$ vs. $29 \%, p=0.002$ ), increased creatinine ( $7 \%$ vs. $18 \%, p=0.09$ ), Grade $3 / 4$ neutropenia ( $23 \%$ vs. $59 \%, p<0.001)$, and febrile neutropenia ( $0 \%$ vs. $3 \%, p=1.00$ ) occurred more frequently in the FOLFIRI group than in the IRI group (Table 3). 
Table 3

Adverse events

\begin{tabular}{|c|c|c|c|c|}
\hline \multirow[t]{4}{*}{ Events } & \multirow{2}{*}{\multicolumn{2}{|c|}{$\begin{array}{l}\text { IRI group } \\
\mathrm{n}=\mathbf{3 1}\end{array}$}} & \multicolumn{2}{|c|}{ FOLFIRI group } \\
\hline & & & $n=76$ & \\
\hline & \multicolumn{2}{|l|}{$\mathrm{n}(\%)$} & \multicolumn{2}{|l|}{$\mathrm{n}(\%)$} \\
\hline & All Grade & Grade $\geq 3$ & All Grade & Grade $\geq 3$ \\
\hline \multicolumn{5}{|l|}{ Hematological } \\
\hline Leukocytopenia & $12(39)$ & $7(23)$ & $44(58)$ & $12(16)$ \\
\hline Neutropenia & $13(42)$ & $7(23)$ & $60(79)$ & $45(59)$ \\
\hline Anemia & $10(32)$ & 0 & $32(42)$ & $3(4)$ \\
\hline Thrombocytopenia & $1(3)$ & 0 & $23(29)$ & $2(3)$ \\
\hline \multicolumn{5}{|l|}{ Nonhematological } \\
\hline Febrile neutropenia & - & 0 & - & $2(3)$ \\
\hline ALT increased & $10(32)$ & $1(3)$ & $24(32)$ & $2(3)$ \\
\hline Creatinine increased & $2(6)$ & 0 & $16(21)$ & 0 \\
\hline Diarrhea & $20(65)$ & $2(6)$ & $43(57)$ & $5(7)$ \\
\hline Fatigue & $12(39)$ & 0 & $34(45)$ & 0 \\
\hline Nausea & $10(32)$ & 0 & $45(59)$ & 0 \\
\hline Decreased appetite & $11(35)$ & 0 & $40(53)$ & $2(3)$ \\
\hline Abdominal pain & $4(13)$ & 0 & $11(14)$ & 0 \\
\hline Alopecia & $12(39)$ & - & $33(43)$ & - \\
\hline Vomiting & $4(13)$ & 0 & $22(29)$ & 0 \\
\hline Constipation & $13(42)$ & $2(6)$ & $25(33)$ & 0 \\
\hline Stomatitis & $4(13)$ & 0 & $27(36)$ & $2(3)$ \\
\hline HFS & 0 & 0 & $10(13)$ & 0 \\
\hline Rash & $5(16)$ & 0 & $9(12)$ & 0 \\
\hline Hiccup & $4(13)$ & 0 & $5(7)$ & 0 \\
\hline Epistaxis & $4(13)$ & 0 & $7(9)$ & 0 \\
\hline
\end{tabular}




\section{Discussion}

In this retrospective study, the PFS and OS of irinotecan plus bevacizumab were similar to that of FOLFIRI plus bevacizumab with a decrease in adverse events. Tumor shrinkage, RR, and DCR of irinotecan plus bevacizumab were also comparable to the FOLFIRI plus bevacizumab regimen, whereas the RDI of irinotecan and bevacizumab did not show a significant difference in both groups. This indicates that irinotecan plus bevacizumab is an alternative treatment for FOLFIRI plus bevacizumab as second-line chemotherapy for patients with $\mathrm{mCRC}$ who are refractory to fluoropyrimidines. To the best of our knowledge, there are no reports that compared the survival outcomes and safety of irinotecan monotherapy to that of FOLFIRI with the same initial dose of irinotecan in combination with bevacizumab.

In the DaVINCI trial, a randomized phase 2 trial in Australia, the efficacy and safety of FOLFIRI was compared with that of irinotecan monotherapy $\left(350 \mathrm{mg} / \mathrm{m}^{2}\right.$ intravenous injection, 3-weekly). The results indicated that FOLFIRI reduced alopecia and diarrhea without compromising the efficacy of OS and PFS (Clarke et al. 2011). However, this study did not evaluate the efficacy and toxicity of continuing 5-FU as second-line chemotherapy, because the initial dose and administration interval of irinotecan were different in both arms. Kuramochi et al. (2017) reported a phase 2 study of irinotecan plus bevacizumab as second-line chemotherapy for patients with $\mathrm{MCRC}$ after first-line chemotherapy containing fluoropyrimidines, oxaliplatin, and bevacizumab. Patients received $150 \mathrm{mg} / \mathrm{m}^{2}$ of irinotecan and 10 $\mathrm{mg} / \mathrm{kg}$ of bevacizumab every two weeks. In this phase 2 study, PFS and OS of patients treated with irinotecan and bevacizumab were 5.7 and 11.8 months, respectively, and adverse events were manageable. No Grade 3/4 oral mucositis and 3.3\% Grade 3/4 fatigue were observed. However, this phase 2 study could not determine whether irinotecan plus bevacizumab was superior to FOLFIRI plus bevacizumab because it was a single-arm study.

FOLFIRI is an inconvenient regimen because it requires 46 hours of continuous infusion and central venous catheter implantation. Therefore, combination regimens of oral capecitabine or S-1 and oxaliplatin (CAPOX or SOX) without central venous catheter implantation are frequently administered as first-line chemotherapy in Japan. The results of this study could contribute a treatment strategy for mCRC without central venous catheter implantation from first-line to later-line treatment, with a difference in the proportion of patients receiving oral fluoropyrimidines ( $84 \%$ vs. $68 \%$, IRI group vs. FOLFIRI group). Moreover, this strategy resulted in a reduction of medical expenses and resources. Moreover, as secondline treatment, other antiangiogenic agents, such as ramucirumab and aflibercept, may be used in combination with FOLFIRI. Our results will be useful when using these drugs in combination with irinotecan (Tabernero et al. 2015; Van Cutsem et al. 2012).

This study had several limitations. First, it was a nonrandomized retrospective study with a small sample size containing differences in patient characteristics. However, we established strict eligibility criteria, such as a CT scan within 28 days before initiation of second-line chemotherapy and the standard initial dose of each drug to obtain more accurate results. In addition, BRAF V600E, which is a poor prognostic 
factor for patients with $\mathrm{mCRC}$, was tested in $96 \%$ and $95 \%$ of patients in the IRI group and FOLFIRI group, respectively (Kayhanian et al. 2018; Tran et al. 2011). Second, interpreting the frequency of neutropenia and diarrhea was difficult because the UDP-glucuronosyltransferase (UGT)1A1 genotype was not evaluated in our study. Approximately $10 \%$ of Japanese patients are homozygous or double heterozygous for UGT1A1 $* 6$ and UGT1A1 $* 28$, which are predictive markers of hematological toxicity for irinotecan (Ando et al. 2000; Minami et al. 2007). This frequency may be relatively small, so we considered that the impact of this limitation to our results was not significant. Finally, the effect of newly approved drugs during the eligibility period on OS was not considered, such as FTD/TPI or regorafenib.

\section{Conclusion}

In conclusion, our study suggests that omitting fluorouracil from FOLFIRI plus bevacizumab as secondline chemotherapy decreases adverse events without affecting treatment efficacy in patients with mCRC who are refractory to fluoropyrimidines in daily practice.

\section{Declarations}

\section{Funding}

This research did not receive any specific grant from funding agencies in the public, commercial, or notfor-profit sectors.

\section{Competing interest}

The authors declare the following conflicts of interest: YM received honorania from Takeda, Merck Bio Pharma, and Taiho. TM received honorania from Takeda, Chudai, Merck Bio Pharma, Taiho, Bayer, Eli Lilly, Yakult Honsha, Sanofi, Daiichi Sankyo, Ono, and Bristol-myers squibb and research funding from MSD, Daiichi Sankyo, Ono, and Novartis. TO received honorania from Ono, Bristol-myers squibb, Taiho, MSD, and Eli Lilly. TN received honorania from Eli Lilly. YN received honorania from Yakult Honsha, Taiho, Eli Lilly, Daiichi Sankyo, and AstraZeneca and research funding from Ono, and Bristol-myers squibb. YN was also a part of the advisory board of Daiichi Sankyo. HB received honorania from Eli Lilly and Taiho and research funding from Ono. HT received honorania from Taiho, Chugai, Takeda, Eli Lilly, Merck Biopharma, and Yakult Honsha. SK received honorania from Bristol-myers squibb, Chugai, Merck Bio Pharma, Daiichi Sankyo, MSD, Ono, Bayer, Taiho, and Esai and research funding from Ono, Taiho, MSD, Nobelpharma, Bristol-myers squibb, Eli Lilly, and Chugai. MT received honorania from EA Pharma. KM received honorania from Ono, Chugai, Takeda, Taiho, Sanofi, Bristol-myers squibb, Eli Lilly, and Bayer; research funding from Solasia Pharma, Merck Serono, Daiichi Sankyo, Parexel International, Pfizer, MSD, Amgen, ONO, Astellas, Sanofi, Taiho, and Esai; and consulting fees from AstraZeneca, Ono, and Amgen. KM was also a part of the advisory boards of Ono, MSD, AstraZeneca, Daiichi Sankyo, and Solasia Pharma.

\section{Ethics approval}


This study was approved by the institutional review board (Aichi Cancer Center Hospital IRB, ref, 2020-1339). The institutional review board approved the waiving of informed consent because of the observational retrospective study design, with an optout opportunity provided on the institution's website.

\section{Data availability}

The datasets generated during and/or analysed during the current study are available from the corresponding author on reasonable request.

\section{Consent to participate}

Informed consent of participants was waived by the ethics committee as a substitute opt-out.

\section{Consent to publish}

Not applicable

\section{Acknowledgements}

The authors would like to thank Enago (www.enago.jp) for the English language review.

\section{Author contributions}

All authors contributed to the study conception and design. Material preparation, data collection and analysis were performed by Yuki Matsubara. The first draft of the manuscript was written by Yuki Matsubara and Toshiki Masuishi, and all authors commented on previous versions of the manuscript. All authors read and approved the final manuscript.

\section{References}

1. Ando Y, Saka H, Ando M, Sawa T, Muro K, Ueoka H, et al (2000) Polymorphisms of UDPglucuronosyltransferase gene and irinotecan toxicity: a pharmacogenetic analysis. Cancer Res 60(24):6921-6926. https://www.ncbi.nlm.nih.gov/pubmed/11156391

2. Clarke SJ, Yip S, Brown C, van Hazel GA, Ransom DT, Goldstein D, et al (2011) Single-agent irinotecan or FOLFIRI as second-line chemotherapy for advanced colorectal cancer; results of a randomised phase II study (DaVINCI) and meta-analysis [corrected]. Eur J Cancer 47(12):1826-1836. https://www.ncbi.nlm.nih.gov/pubmed/21665462

3. Kayhanian H, Goode E, Sclafani F, Ang JE, Gerlinger M, Gonzalez de Castro D, et al (2018) Treatment and survival outcome of BRAF-mutated metastatic colorectal cancer: a retrospective matched casecontrol study. Clin Colorectal Cancer 17(1):e69-e76. https://www.ncbi.nlm.nih.gov/pubmed/29129559

4. Kuramochi H, Ando M, Itabashi M, Nakajima G, Kawakami K, Hamano M, et al (2017) Phase Il study of bevacizumab and irinotecan as second-line therapy for patients with metastatic colorectal cancer 
previously treated with fluoropyrimidines, oxaliplatin, and bevacizumab. Cancer Chemother Pharmacol 79(3):579-585. https://www.ncbi.nlm.nih.gov/pubmed/28213683

5. Minami H, Sai K, Saeki M, Saito Y, Ozawa S, Suzuki K, et al (2007) Irinotecan pharmacokinetics/pharmacodynamics and UGT1A genetic polymorphisms in Japanese: roles of UGT1A1*6 and *28. Pharmacogenet Genomics 17(7):497-504. https://www.ncbi.nlm.nih.gov/pubmed/17558305

6. Rothenberg ML, Oza AM, Bigelow RH, Berlin JD, Marshall JL, Ramanathan RK, et al (2003) Superiority of oxaliplatin and fluorouracil-leucovorin compared with either therapy alone in patients with progressive colorectal cancer after irinotecan and fluorouracil-leucovorin: interim results of a phase III trial. J Clin Oncol 21(11):2059-2069. https://www.ncbi.nlm.nih.gov/pubmed/12775730

7. Tabernero J, Yoshino T, Cohn AL, Obermannova R, Bodoky G, Garcia-Carbonero R, et al (2015) Ramucirumab versus placebo in combination with second-line FOLFIRI in patients with metastatic colorectal carcinoma that progressed during or after first-line therapy with bevacizumab, oxaliplatin, and a fluoropyrimidine (RAISE): a randomised, double-blind, multicentre, phase 3 study. Lancet Oncol 16(5):499-508. https://www.ncbi.nlm.nih.gov/pubmed/25877855

8. Tournigand C, André T, Achille E, Lledo G, Flesh M, Mery-Mignard D, et al (2004) FOLFIRI followed by FOLFOX6 or the reverse sequence in advanced colorectal cancer: a randomized GERCOR study. $J$ Clin Oncol 22(2):229-237. https://www.ncbi.nlm.nih.gov/pubmed/14657227

9. Tran B, Kopetz S, Tie J, Gibbs P, Jiang ZQ, Lieu CH, et al (2011) Impact of BRAF mutation and microsatellite instability on the pattern of metastatic spread and prognosis in metastatic colorectal cancer. Cancer 117(20):4623-4632. https://www.ncbi.nlm.nih.gov/pubmed/21456008

10. Van Cutsem E, Tabernero J, Lakomy R, Prenen H, Prausová J, Macarulla T, et al (2012) Addition of aflibercept to fluorouracil, leucovorin, and irinotecan improves survival in a phase III randomized trial in patients with metastatic colorectal cancer previously treated with an oxaliplatin-based regimen. $J$ Clin Oncol 30(28):3499-3506. https://www.ncbi.nlm.nih.gov/pubmed/22949147

\section{Figures}



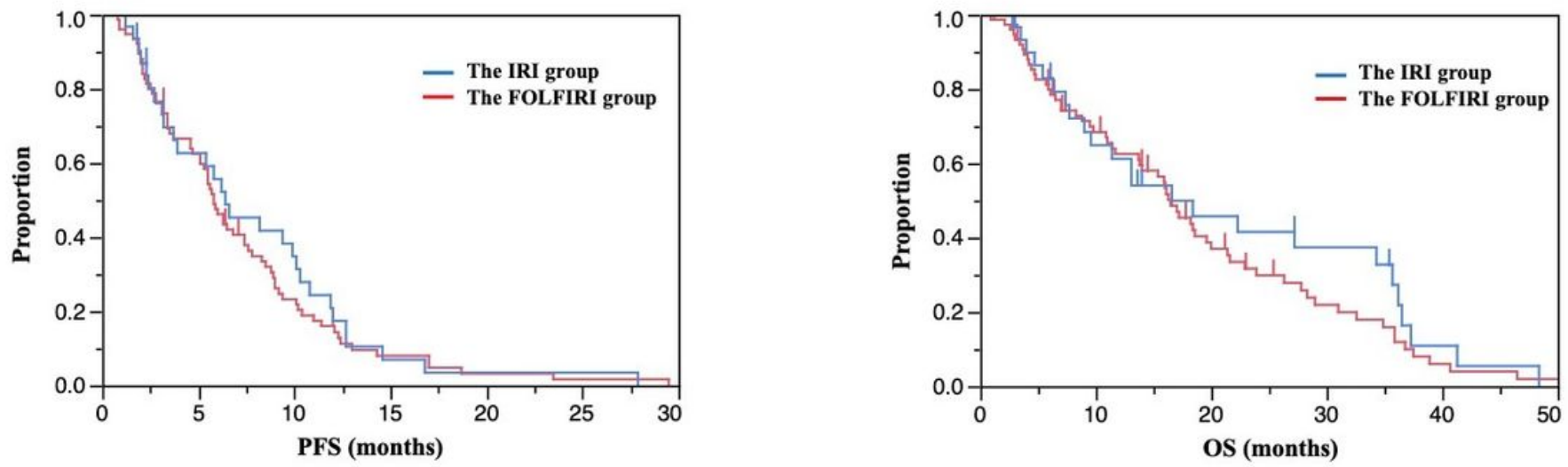

Number at risk

$\begin{array}{llllllll}\text { IRI group } & 31 & 18 & 10 & 2 & 1 & 1 & 0\end{array}$

Number at risk

$\begin{array}{lllllll}\text { IRI group } & 31 & 18 & 11 & 8 & 2 & 0 \\ \text { FOLFIRI group } & 76 & 47 & 23 & 11 & 3 & 1\end{array}$

\section{Figure 1}

\section{Overall survival and progression-free survival}

The median follow-up time was 13.1 (range, 1.2-48.4) months in the IRI group and 14.3 (range, 0.946.5) months in the FOLFIRI group. Median progression-free survival (PFS) was 6.4 months in the IRI group and 5.8 months in the FOLFIRI group [hazard ratio (HR), 0.90; 95\% confidence interval (Cl), 0.571.38; $p=0.64$; adjusted $\mathrm{HR}, 0.82 ; 95 \% \mathrm{Cl}, 0.50-1.34, p=0.44]$. PFS was adjusted for previous colorectal resection, the number of metastatic sites, Eastern Cooperative Oncology Group Performance Status (ECOG PS), liver metastasis, and lactate dehydrogenase levels (LDH). Median overall survival (OS) was 16.6 months in the FOLFIRI group versus 16.5 months in the FOLFIRI group (HR, $0.83 ; 95 \% \mathrm{Cl}, 0.51-1.32$; $p=0.44 ;$ aHR, $1.01 ; 95 \% \mathrm{Cl}, 0.59-1.69 ; p=0.97)$. OS was adjusted for prior colectomy, the number of metastatic sites, liver metastasis, peritoneal metastasis, and LDH levels
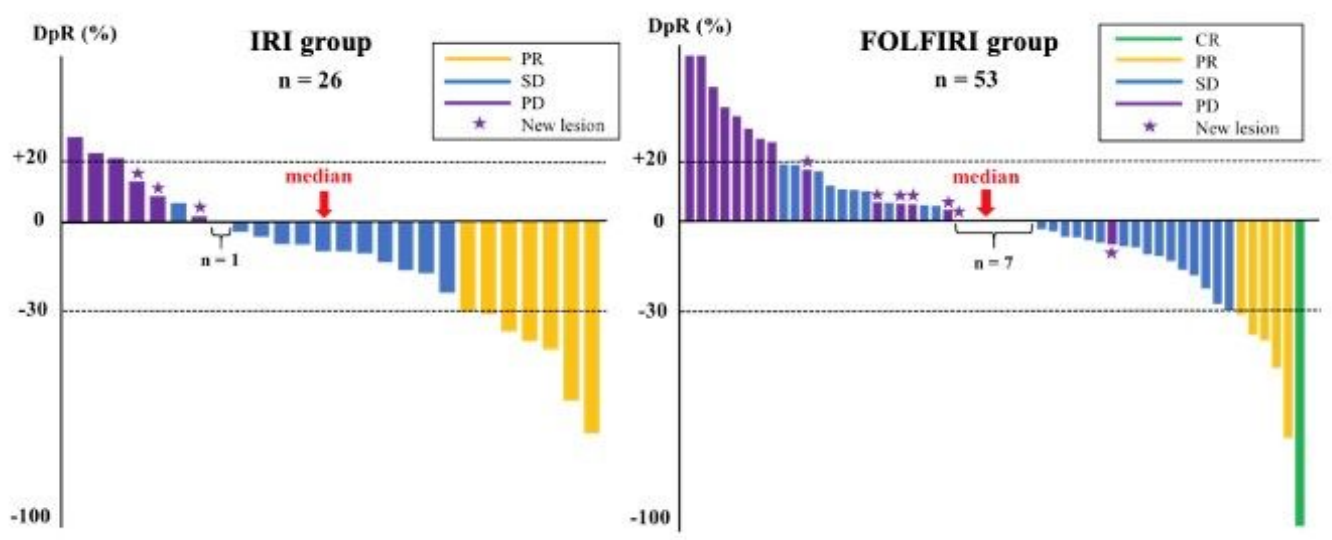

\begin{tabular}{ccc}
\hline & $\begin{array}{c}\text { IRI group } \\
\mathbf{n}=\mathbf{2 7}\end{array}$ & $\begin{array}{c}\text { FOLFIRI group } \\
\mathbf{n}=\mathbf{5 3}\end{array}$ \\
\hline RR & $26 \%$ & $11 \%$ \\
DCR & $74 \%$ & $72 \%$ \\
\hline \multicolumn{3}{c}{ Best Response } \\
n (\%) \\
\hline CR & $0(0)$ & $1(2)$ \\
PR & $7(26)$ & $5(9)$ \\
SD & $13(48)$ & $32(60)$ \\
PD & $6(22)$ & $15(28)$ \\
NE & $1(4)$ & $0(0)$ \\
\hline
\end{tabular}

Figure 2

Best response 
Among 107 patients, 27 patients in the IRI group and 53 patients in the FOLFIRI group had measurable lesions. The deepness of response (DpR) presented as a waterfall plot was defined as the rate of tumor shrinkage from baseline CT. In the IRI group, the response rate (RR) and disease control rate (DCR) were $26 \%$ and $74 \%$, respectively. In the FOLFIRI group, RR and DCR were $11 \%$ and $72 \%$, respectively.

Abbreviation; $\mathrm{CR}$, complete response; $\mathrm{PR}$, partial response; SD, stable disease; $\mathrm{PD}$, progressive disease; $\mathrm{NE}$, not evaluated 